\title{
Current Use of Position-Sensitive Proportional Counters for Small-Angle X-ray Scattering and Diffraction
}

\author{
BY Y. Dupont \\ Max-Planck Institut für Medizinische Forschung, Heidelberg, Germany (BRD) \\ A. Gabriel \\ Institut des Sciences Nucléaires, Grenoble, France \\ AND M. Chabre AND V. Luzzati \\ Centre de Génétique Moléculaire, C.N.R.S., Gif-sur-Yvette, France
}

Linear position-sensitive proportional counters have been developed for small-angle X-ray scattering from the original idea of Borskowsky and Kopp [Dupont, Y., Gabriel, A., Chabre, M., Gulik-Krzywicki, T., \& Shechter, E. (1972). Nature, Lond. 238, 331]. They have been currently used for more than one year in a biophysical laboratory. The most interesting property of this detector is a considerable shortening of the exposure time needed to record the data. The experimental set-up is described. The advantages and limitations of this type of detector compared with the standard film or counter detection are discussed. Experimental results involving the diffuse small-angle scattering of serum lipoproteins and the sharp small-angle reflexions of rod outer segments of the intact retina are given as examples.

J. Appl. Cryst. (1974). 7, 117

An Report on The Application of Synchrotron Radiation to Low-Angle Scattering

\author{
By J. BarRington Leigh and G. Rosenbaum \\ Max Planck Institut für medizinische Forschung, Heidelberg and DESY (Deutsches Elektron Synchrotron), \\ Hamburg, Germany $(B R D)^{*}$
}

(Received 31 October 1973; accepted 31 October 1973)

\begin{abstract}
The use of synchrotron radiation as an intense X-ray source for biological diffraction has only very recently been promoted into a long-term experimental programme. Here we report on some general and some detailed aspects of this development. In the case of small-angle studies on weakly scattering specimens at high time resolution, e.g. stroboscopic diffraction studies on muscle, the lack of X-ray sources of considerably higher brightness than from conventional X-ray generators has become limiting. As it has become technically impossible to pursue the development of rotating-anode tubes much further, the use of an electron synchrotron or storage ring with 100 to 1000 times higher brightness opens up the possibility not only of collecting much improved data but also of whole new ranges of experiments. A brief description is presented of the low-angle diffraction equipment currently in use at the synchrotron in Hamburg.
\end{abstract}

\section{Introduction}

The topics to be discussed in this short review concern both the present and future use of synchrotron radiation as a very bright $\mathrm{X}$-ray source for low-angle scattering. The authors have been concerned with establishing an X-ray diffraction facility in a new bunker on the $7.5 \mathrm{GeV}$ electron synchrotron ring DESY at

* Authors' present address: European Molecular Biology Laboratory, 69 Heidelberg 1, Postfach 2027, Germany (BRD).
Hamburg where some experience has been gained in the low-angle diffraction from insect flight muscle. The properties of synchrotron light sources have been reviewed elsewhere (Godwin, 1970) and a detailed discussion of the optics of a synchrotron diffraction arrangement is also available (Barrington Leigh, Holmes \& Rosenbaum, 1972). In addition to summarizing the nature of X-ray sources, the technical considerations for designing suitable diffraction equipment are compiled together with short descriptions of the apparatus presently in use. 